\section{Gérard Zalcman}

Violaine Closson

Nicole Honoré Birgitta Olofsson Armand Tavitian

\section{ADRESSE}

(;. Zalcman: chef de clinique-assistant, service de pneumologie, hôpital Saint-Louis. V. ('losson: ancien interne des hopitaux de P'aris. $N$. Honoré: ingénieur d'études, université l'uris-VII; B. Olofsson : directeur de recherche au (inrs. A. Tavitian: direcleur de recherche is I'Inserm. Inserm U.248, Signalisation intracellulaire et oncogenèse, faculté de médecine Lariboisière-Saint-I.ouis, 10, avenue de Verdun, 75010 Paris, France.

\title{
Participation de la cascade des protéines Rho à la régulation du cytosquelette: rôle possible dans les mécanismes d'oncogenèse
}

Les outils de la biologie cellulaire ont permis de disséquer les voies de transmission du signal passant par les protéines Rho (en particulier les protéines Rho, Rac et Cdc42). Ce sont des membres de la superfamille des GTPases Ras, impliquées dans le remodelage permanent du cytosquelette d'actine provoqué par la bradykinine ou les facteurs de croissance. Elles jouent, de ce fait, un rôle central dans la vie cellulaire, contrôlant finement la motilité, la phagocytose, la sécrétion polarisée. Est-ce par leur action sur le cytosquelette d'actine qu'elles sont impliquées dans la division cellulaire? Quant à leur participation aux processus de carcinogenèse, elle reste controversée; cependant, un couplage a été récemment décrit entre la voie de signalisation passant par Ras et la cascade des protéines Rho.

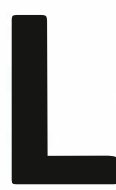

es protéines de la famille Rho (Ras homologue), membres de la superfamille des GTPases Ras, ont été l'objet d'un intérêt croissant au cours des trois dernières années. Comme toutes les protéines apparentées à Ras, les protéines Rho sont capables de lier de façon réversible les nucléotides guanyliques. Ces protéines existent en effet sous deux formes: une forme inactive liée au GIDP est activée par un changement conformationnel induit par la fixation de GTP en lieu et place du
GDP. Ce cycle est réglé : (1) par leur faible activité GTPasique intrinsèque; (2) par des protéines GAP (GTPase activating proteins) [1] qui stimulent cette activité GTPasique, permettant le retour à une forme inactive liée au GDP; (3) par des protéines inhibant l'échange GDP $\rightarrow$ GTP, ou inhibant l'action des protéines GAP, les GDI (GI)P-dissociation inhibitors); (4) par des protéines stimulant cet échange et permettant l'activation des protéines Rho, les GDS ( GI)P-dissociation stimulators ou facteurs d'échange) [2]. 


\section{RÉFÉRENCES}

1. Fort P, Vincent S. Transduction du signal mitogène, cytosquelette et petites protéines $\mathrm{G}$ : vers un réseau de protéines GAP? médecine/sciences 1993; 9: 59-65.

2. Galland F, Birnbaum D. Le proto-oncogène $\mathrm{mcf} 2 / \mathrm{dbl}$ et les facteurs d'échange GDP-GTP. médecine/sriences 1992; 8: 819-26.

3. Dallery E, Galiègue-Zouitina S, Collynd'Hooghe M, et al. TTF, a gene encoding a novel small (; protein, fuses to the lymphoma-associated $\mathrm{IAZ3}$ gene by $\mathrm{t}(3 ; 4)$ chromosomal translocation. Oncogene 1995; 10: 2171-8.

4. Chardin P, Bocquet P, Madaule P, Popoff MR, Rubin EJ, Gill DM. The mammalian (: protein RhoC is ADP-ribosylated by Clostridium botulinum exoenzyme $\mathrm{C} 3$ and affects actin microfilaments in Vero cells. $E M B O J$ $1989 ; 8: 1087-92$.

5. Munemitsu S, Innis MA, Clark R, McCormick F, Ullrich A, Polakis P. Molecular cloning and expression of a G25K cDNA, the human homolog of the yeast cell cycle gene CDC42. Mol Cipll Biol 1990; 10: 5977-82.

6. Ridley AJ, Hall A. The small GTP-binding protein Rho regulates the assembly of focal adhesions and actin stress fibers in response to growth factor. (ill 1992; $70: 389-99$.

7. Kozma R, Ahmed S, Best A, Lim I. The Ras-related Cdc42Hs and bradykinin pro mote formation of peripheral actin microspikes and filopodia in Swiss 3T3 fibroblasts. Mol Cell Biol 1995; 15 : 1942-52.

8. Nobes (AD, Hall A. Rho, Rac, and Cdc42 GTPases regulate the assembly of multimolecular focal complexes associated with actin stress fibers, lamellipodia, and filopodia. Cell 1995; $81: 53-62$.

9. Ridley AI, Paterson HF, Johnston CL. Diekmann D, Hall A. The small GTP-binding protein Rac regulates growth factorinduced membrane ruffling. Cell 1992; 70 : 401-10.

10. Nobes CD, Hawkins P, Stephens I, Hall A. Activation of the small GTP-binding proteins Rho and Rac by growth factor receptors. I Cell Sici 1995; 108 : 225-33.

11. Diekman I), Abo A, Johnston C, Segal AW, Hall A. Interaction of Rac with $\mathrm{p}^{6} 7^{\text {phux }}$ and regulation of phagocytic NADPH oxidase activity. Science 1994; 265: 531-3.

12. Peppelenbosch MP, Qiu RG, de VriesSmits AMM, et al. Rac mediates growth factor-induced arachidonic acid release. Ciell

Chez l'homme, neuf protéines Rho ont été caractérisées à ce jour: RhoA, RhoB et RhoC, analogues à plus de $85 \%$ entre elles et à $35 \%$ avec les protéines Ras; Racl, Rac 2 et RhoG; Cdc42Hs et TCl0; TTF, codée par un gène transloqué sur le chromosome 3, dans certains lymphomes non hodgkiniens [3].

La fonction des gènes $R H O$ est restée très longtemps mystérieuse. Des arguments indirects ont cependant très vite impliqué Rho A et Rho C dans la régulation du cytosquelette d'actine. Ces protéines sont, en effet, substrats d'enzymes bactériennes, comme la toxine botulinique C3, qui les inactive en catalysant la fixation d'un AIDPribose sur leur site effecteur: la microinjection d'exo-enzyme C3 dans des cellules fibroblastiques induit une réorganisation rapide du cytosquelette avec dépolymérisation des filaments d'actine, et acquisition d'un phénotype arrondi [4]. (DC:42Hs est, quant à lui, l'analogue humain d'un gène de levure, caractérisé comme un élément important de la signalisation intracellulaire, qui permet le bourgeonnement de la levure à la suite de réarrangements locaux des filaments d'actine au niveau du site de bourgeonnement [5].

\section{Protéines Rho et régulation du cytosquelette d'actine}

Des travaux récents ont permis une meilleure compréhension de la régulation fonctionnelle des protéines Rho en démontrant l'existence d'une véritable cascade de signalisation, aboutissant à un contrôle très fin du remodelage du cytosquelette d'actine au cours de processus aussi variés que la motilité cellulaire, la phagocytose ou la sécrétion polarisée $\left(\mathrm{m} / \mathrm{s} n^{\circ} 7\right.$, vol. $\left.11, \mathrm{p} .1045\right)$. Après micro-injection dans des cellules fibroblastiques Swiss 3T3 de protéines Rho purifiées ou de vecteurs codant pour des protéines Rho, Racl ou Cdc42Hs, étiquetées par un épitope Myc, sauvages ou mutantes, activées (substitution G14V pour Rho ou G12V pour Racl et (:dc42Hs) ou dominantes négatives (substitution Sl7N), le marquage par immunofluorescence au moyen d'anticorps spécifiques de plusieurs composants protéiques du cytosquelette (actine, vinculine, taline, paxilline) a permis de préciser l'action spécifique de chacune de ces protéines Rho, en réponse à différents facteurs de croissance [6-10].

Ainsi, il apparaît clairement que la bradykinine induit l'activation de Cdc42Hs qui, sous sa forme GTP, est responsable de la formation de petites excroissances ou microspicules de $25 \mu \mathrm{m}$ de long à la surface de la cellule, les filopodes, dont la structure est constituée de complexes multiprotéiques associant actine, vinculine et paxilline dans un environnement enrichi en p $125^{r+k}$, la kinase associée aux plaques d'adhérence [7, 8]. La formation de ces filopodes s'accompagne d'une rétraction du corps cellulaire due à des fibres d'actine "de rétraction". Secondairement, Cdc42Hs liée au GTP semble entraîner l'activation de Racl. Cette activation peut être aussi obtenue après stimulation cellulaire par le PI)GF (platelet-derived growth factor), l'EGF (epidermal growth factor) ou l'insuline $[8,9]$, ces facteurs de croissance exerçant leurs effets par l'intermédiaire de la phosphatidylinositol 3-kinase (PI3K) [10]. Racl activé (lié au GTP) est alors responsable de la formation de replis de la surface cellulaire (mefles) et de véritables lamellipodes impliqués dans les mécanismes de reptation cellulaire [9]. De telles structures cellulaires sont aussi observées dans les cellules phagocytaires, dans lesquelles Rac s'associe à la protéine p67 phax pour régler l'activité NAIPH-oxydase membranaire, cruciale dans le phénomène de phagocytose [11]. Ces lamellipodes sont liés à une polymérisation locale sous-membranaire de l'actine avec formation de petits agrégats de vinculine et de paxilline au niveau du front de progression du lamellipode [8]. Ces complexes focaux multimoléculaires sont éphémères, témoignant d'une structure dynamique. La formation de filopodes et de lamellipodes apparaît, de plus, parfaitement coordonnée dans le temps et dans l'espace.

Enfin, Racl-GTP est susceptible d'activer RhoA, ainsi que le ferait une stimulation cellulaire par l'acide lysophosphatidique (LPA) ou par la bombésine, facteur de croissance autocrine de certaines lignées tumorales [6]. Cette activation pourrait

$1995 ; 81: 849-56$ 


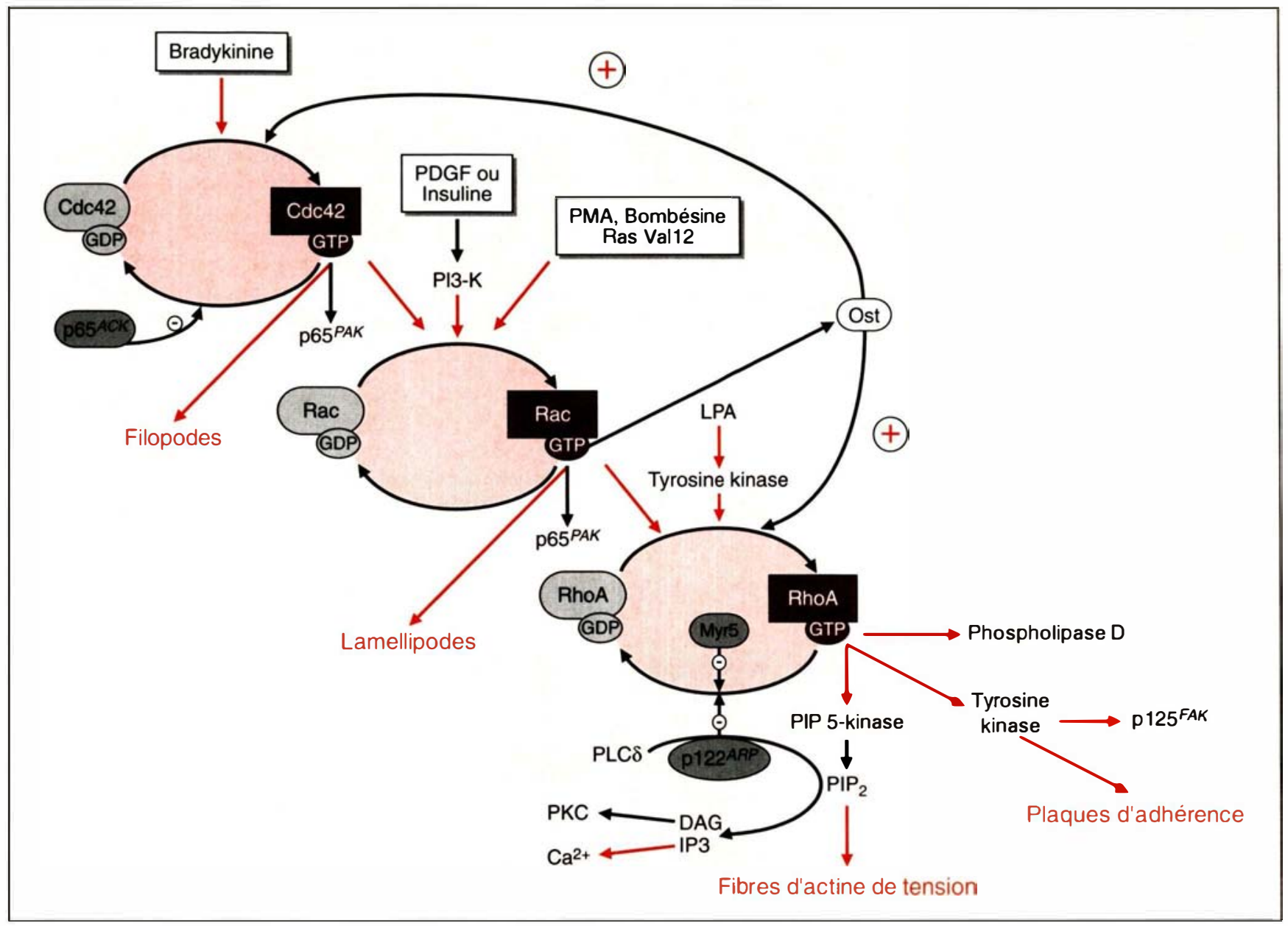

Figure 1. La cascade de signalisation des protéines Rho. Les flèches rouges indiquent l'existence d'un lien fonctionnel (direct ou indirect). Les flèches noires indiquent l'existence d'interactions physiques (in vitro ou in vivo). La cascade des Rho pourrait être couplée à la voie de signalisation Ras $\rightarrow$ Raf $\rightarrow$ Map kinase par l'intermédiaire de protéines multifonctionnelles à activité GAP ou facteur d'échange vis-à-vis des Rho et/ou de Ras, liant la régulation du cytosquelette à la prolifération cellulaire. PI3-K: phosphatidylinositol 3-kinase; LPA: acide lysophosphatidique; PMA: phorbol 12-myristate 13-acétate; PKC: protéine kinase C; DAG: diacylglycérol; IP3: inositol trisphosphate; PIP 5-kinase: Ptd-Ins 4,5- $P_{2}$ kinase; PIP2: Ptd-Ins 4,5- $P_{2} ; P L C \delta$ : phospholipase $C \delta$; FAK: focal adhesion kinase 1 kinase des plaques $d^{\prime}$ adhérence); PAK: $p 21$ activated kinase ; $A C K$ : Cdc42Hs-associated kinase ; ARP: adapter for Rho and PLC $\delta$; Myr5: 5th unconventional myosin from rat. (D'après [6-10, 16-21, 39]).

être relayée par la libération d'acide arachidonique et de ses dérivés, les leucotriènes [12]. RhoA-GTP induit alors la formation de plaques d'adhérence et de faisceaux de fibres d'actine de tension émanant de ces plaques [1]. Ces structures permettent à la cellule de s'ancrer au support (matrice extracellulaire) sur des points d'appui, notamment lors de la reptation cellulaire.

$\mathrm{m} / \mathrm{s} n^{\circ} I I$, vol. 11 , novembre 95

\section{Voies de transduction des signaux passant par les Rho}

Les effecteurs de ces différentes protéines Rho ne sont qu'en partie connus et pourraient varier selon le type cellulaire considéré, ainsi que le montre la présence de $\mathrm{p} 67^{\text {thax }}$, partenaire de Rac, dans les seules cellules phagocytaires [11].
L'un de ces effecteurs, l'oncoprotéine Ost, interagit spécifiquement avec Rac-GTP alors qu'elle possède une activité d'échange GDP-GTP pour RhoA et Cdc42Hs, suggérant une boucle d'amplification positive dans la cascade Rho [13]. D'autres facteurs d'échange pourraient régler les différents éléments de cette voie de signalisation et les coupler à d'autres voies, ainsi que le suggèrent certaines 


\section{RÉFÉRENCES}

13. Horii Y, Beeler JF, Sakaguchi K, Tachibana M, Miki T. A novel oncogene, ost, encodes a guanine nucleotide exchange factor that potentially links Rho and Rac signaling pathways. $E M B O J 1994 ; 13: 4776-86$.

14. Plessis A, Camonis JH. Le système double-hybride, mode d'emploi. méderine/sriences 1994; 10: I-IX.

15. Manser E, Leung T, Salihudin H, Zhao Z, Lim L. A brain serine/threonine protein kinase activated by Cdc42 and Racl. Nature 1994; 367: 40-6.

16. Manser E, Leung T, Salihuddin H, Tan L, Lim L. A non-receptor tyrosine kinase that inhibits the GTPase activity of p2lcde42. Nature 1993; 363: 364-7.

17. Ridley AJ, Hall A. Signal transduction pathways regulating rho-mediated stress fibre formation: requirement for a tyrosine kinase. LMBO / 1994; 13: 2600-10.

18. Chong LD, Traynor-Kaplan A, Bokoch GM, Schwart\% MA. The small GTP-binding protein Rho regulates a phosphatidylinositol 4-phosphate 5-kinase in mammalian cells. Cill 1994:79:507-13.

19. Homma $Y$, Emori Y. $\Lambda$ dual functional signal mediator showing RhoGAP and phospholipase ( - d stimulating activities. $l M B O J$ $1995 ; 14: 286-91$

20. Reinhard J, Scheel AA, Diekman D, Hall A, Ruppert C, Bähler M. A novel type of myosin implicated in signalling by Rho family (;TPases. EMBO J 1995; 14:697-704.

21. Malcom KC:, Ross AH, Qiu RC; Symons $M$, Exton JH. Activation of rat liver phospholipase $D$ by the small (iTP-binding protein RhoA. I Biol chem 1994; 269: 25951-4.

22. Yamamoto M, Marui N, Sakai T, et al. ADP-ribosylation of the rho $A$ gene product by bolulinum (:3 exoenzyme causes Swiss 3 T3 cells to accumulate in the 6 il phase of the coll cycle. Oncogene 1993; 8 : 1449-55.

23. Adamson P, Marshall C.J, Hall A, Til brook PA. Post-translational modifications of p21 Rho proteins. J Biol Chem 1992; 267: 20033-8.

24. Adamson P, Paterson II, Hall A. Intracellular localization of the p21Rho protein. J Cell Biol 1992; 119:617-27.

25. Zalcman G, Closson V, Linarès-Cruz G, et al. Regulation of Ras-related RhoB protein expression during the cell cycle. Onro données préliminaires issues de la quête de partenaires protéiques des Rho par la technique de clonage par interaction fonctionnelle in vivo dite "du double-hybride" $\left(m / s \quad n^{\circ} 2\right.$, vol. 10, p. 206) [14].

Parmi les autres candidats partenaires, plusieurs kinases se partagent la vedette. Une sérine-thréonine kinase, PAK, a été caractérisée par son interaction in vitro avec $\mathrm{Rac}$ et Cdc42Hs [15]. Ces deux petites protéines $G$ sont capables d'induire l'auto-phosphorylation de PAK, mais la fonction de cette dernière reste à ce jour inconnue. Cependant, sa grande analogie avec la kinase STE20 de $S$. cerevisice impliquée dans la transmission du signal en aval du récepteur des phéromones couplé à une protéine $G$ hétérotrimérique rend compte de la grande conservation des voies de signalisation au cours de l'évolution phylogénétique.

Une deuxième kinase, la tyrosine kinase ACK, dont le domaine catalytique est très proche de celui de pl25 ${ }^{\text {i:tK }}$, s'avère elle aussi interagir in vitro avec Cdc42Hs. Elle inhiberait l'activité GTPasique intrinsèque de cette protéine Rho, la maintenant sous une forme active, liée au GTP [16].

L'implication d'une ou plusieurs autres kinases dans cette cascade de signalisation est démontrée par l'utilisation d'inhibiteurs, comme la génistéine, la staurosporine ou la tyrphostine. La première inhibe la formation de plaques d'adhérence et des fibres d'actine de tension qui en émanent, induites par l'injection de RhoA activée ou par le LPA; la seconde n'inhibe que la formation de plaques d'adhérence, sans inhiber la polymérisation de l'actine, ce qui indique l'existence en aval de RhoA, de deux voies de signalisation divergentes [17]. Ainsi, une tyrosine kinase (inhibée par la génistéine) est activée par RhoA cependant qu'une autre tyrosine kinase (inhibée par la tyrphostine) pourrait se situer juste en aval du LPA, la tyrphostine ne pouvant inhiber l'effet de la microinjection de protéine RhoA G14V [10]. En outre, l'orthovanadate, inhibiteur des phosphotyrosine phosphatases semble, lui aussi, susceptible de stimuler la formation de fibres d'actine de tension, effet bloqué par l'inactivation préalable de RhoA par micro-injection d'exo-enzyme C3. Il existe donc en amont de RhoA un cycle tyrosine kinase/tyrosine phosphatase.

Il semble, par ailleurs, désormais établi que RhoA, sous sa forme GTP, stimule l'activité d'une phosphatidylinositol 4,5-bisphosphate kinase (Ptd-Ins 4,5- $\mathrm{P}_{2}$ kinase), entraînant la libération de Ptd-Ins 4,5- $\mathrm{P}_{2}$ susceptible de régler la fonction de protéines se liant à l'actine, comme la gelsoline, l' $\alpha$-actinine, la profilline, contenues dans les plaques d'adhérence [18]. L'augmentation locale de la concentration en Ptd-Ins $4,5-\mathrm{P}_{2}$ induirait la dissociation entre les molécules de profilline et les monomères d'actine (actine $G$ ), et détacherait la gelsoline des extrémités d'oligomères d'actine (actine F), permettant une polymérisation locale de l'actine. La caractérisation récente d'une protéine de $122 \mathrm{kD}$ a exerçant une activité GAP sur RhoA et s'associant à la phospholipase $\mathrm{C} \delta$ pour l'activer et induire l'hydrolyse du Ptdlns 4,5- $\mathrm{P}_{2}$ permet de décrire une boucle de rétrocontrôle de cette voie de signalisation ou, alternativement, la suite de la transmission du signal [19] : l'hydrolyse du Ptd-Ins 4,5- $\mathrm{P}_{2}$ engendre du diacylglycérol et de l'inositol 1,4,5-trisphosphate, le premier activant une protéine kinase $\mathrm{C}$, le second activant les systèmes dépendants du calcium. Une autre boucle de rétro-contrôle pourrait être représentée par un nouveau type de myosine, Myr 5, qui interagit avec l'actine $\mathrm{F}$, mais possède aussi un domaine GAP capable de stimuler l'activité GTPasique de RhoA, Cdc42Hs, voire de $\mathrm{Racl}$, favorisant leur retour à une conformation inactive, liée au GIDP [20]. Enfin, RhoA pourrait aussi activer (directement ou indirectement) une phospholipase D membranaire catalysant l'hydrolyse de phosphatidylcholine en acide phosphatidique et choline, en réponse à une stimulation par des facteurs de croissance. L'acide phosphatidique pourrait constituer un second messager impliqué dans des voies aval de signalisation [21].

\section{Protéines Rho et cycle cellulaire}

Une implication directe des protéines Rho dans le déroulement du cycle cellulaire a été suggérée par 
l'action de la toxine C3 sur des cellules Swiss 3T3: ces cellules sont bloquées dans leur cycle cellulaire en phase (;1, l'exo-enzyme C3 ADPribosylant une protéine identifiée comme étant RhoA, seule Rho à être détectée dans ces cellules en phase exponentielle, et de ce fait "accusée" de jouer un rôle critique dans la transition (il-S [22].

Cependant, ce rôle dans la division cellulaire pourrait plutôt être le fait de la protéine RhoB, dont la localisation (endosomes précoces et compartiment pré-lysosomial) diffère de celle de RhoA et RhoC (cytosolique et, dans une moindre mesure, membranaire), vraisemblablement en relation avec des modifications post-traductionnelles différentes [23-25]. En effet, rhoB est le premier gène de la superfamille ras dont on a démontré qu'il appartenait à la catégorie des gènes immédiats-précoces. Son expression est rapidement induite lorsque des cellules fibroblastiques, épithéliales ou neuro-endocrines passent d'un état de "quiescence " à un état de prolifération sous l'effet de facteurs de croisssance (EGF, PD(;F, insuline...) [25, 26]. Nous avons, par ailleurs, montré que la protéine RhoB n'était que très difficilement détectable dans des cellules en phase exponentielle puisque spécifiquement exprimée à partir de la transition (il-S, puis au cours de la phase $\mathrm{S}$ du cycle cellulaire [25]. Ces données pourraient rendre compte d'un rôle de $\mathrm{RhoB}$ dans la réorganisation du cytosquelette observée au cours du cycle cellulaire. D'autres arguments indirects, comme la localisation vésiculaire de RhoB associée aux effets morphologiques de sa surexpression, (contraction du corps cellulaire: rhophenotype) $[6,25]$, pourraient suggérer un rôle dans le transport ou le recyclage de constituants cytosquelettiques nécessaires au déroulement de la phase $\mathrm{S}$, voire de la mitose.

\section{Protéines Rho et carcinogenèse}

La participation des protéines Rho dans les processus de carcinogenèse reste controversée. La surexpression du gène rhoA normal dans des lignées fibroblastiques induit un phénotype partiellement transformé (moindre dépendance vis-à-vis du sérum, diminution de l'inhibition de contact, tumorigénicité tardive chez la souris nude) [27]. ('ependant, les mutations 14 ou 64 de rhoA (équivalentes des mutations 12 et 61 de Haras) ne permettent pas l'obtention de foyers transformés. L'étude du gène rhoA, localisé sur le bras court du chromosome 3, en 3p21, n'a pas mis en évidence de mutation activante, ni de différence d'expression dans des lignées ou tumeurs du sein, du poumon, du rein, qui présentent pourtant très fréquemment des délétions ou des réarrangements de cette région chromosomique [28].

La surexpression du gène rho d'Aplysie dans des cellules fibroblastiques murines, sous sa forme normale ou avec la substitution (; $14 \mathrm{~V}$ activante a, en revanche, permis d'obtenir des "pseudo-foyers" de transformation et des tumeurs chez la souris nude (en 3 à 4 semaines pour rho muté, 7 à 8 semaines pour rho normal, contre 10 à 14 jours pour des cellules transfectées par H-ras) [29]. A partir de ces tumeurs, des lignées de fibrosarcomes bien différenciés ont été obtenues. Lorsque ces fibroblastes transformés sont privés de sérum, on observe l'exacerbation d'un phénomène d'apoptose identique à ce qui est décrit après transfection des gènes $E 1 A$ ou c-myc, et une susceptibilité accrue aux substances cytotoxiques comme le ci.-platine [30].

Ces derniers faits expérimentaux sont à rapprocher de l'inhibition complète de croissance lorsqu'un rho(iI)I (dont le produit inactive les protéines Rho en les complexant) est surexprimé dans $S$. cerevisicie, suggérant un rôle des protéines Rho dans la multiplication cellulaire [31].

Enfin, la surexpression de racl, muté pour donner un produit activé par la substitution G12V, permet de transformer des cellules fibroblastiques Ratl et ainsi d'obtenir des lignées fortement tumorigènes pour la souris nude (m/s $n^{\circ} 7, v o l .11, p$. 1045) [32]. A l'inverse, la transfection d'un mutant dominant négatif de racl $(\operatorname{rac} 17 \mathrm{~N})$ rend des cellules NIH3T3 résistantes à la transformation induite par la surexpression d'un gène ras activé (ras Gl2V), suggérant que Rac agit en aval de Ras.

En outre, Rac semble se situer sur une voie de signalisation parallèle à celle des MAP kinases. En effet, la transfection de ras $17 \mathrm{~N}$ ne peut prévenir la transformation induite par la surexpression d'un gène raf codant pour une protéine ciblée à la membrane par une séquence $\mathrm{C}$-terminale (CAAX) de ciblage membranaire [33], un tel ciblage mimant l'action de Ras et permettant de stimuler la voie des MAP kinases. De même Rac G12V agit en synergie avec RafCAAX pour transformer des cellules fibroblastiques. Ainsi, Ras pourrait-il activer Rac indépendamment de Raf: le schéma classique de la transformation cellulaire relayée par la voie Ras $\rightarrow$ Raf $\rightarrow$ MAP-kinase se double désormais d'une deuxième voie $R a s$ $\rightarrow$ Rac.

C'est finalement la constatation que plusieurs oncogènes récemment caractérisés possèdent un domaine de type "facteur d'échange" pour les protéines Rho qui renforce le concept d'un rôle clé des Rho dans les phénomènes de transformation. Ainsi l'oncogène $\boldsymbol{l}) b l$ est-il capable de stimuler l'échange guanylique de Cdc42Hs et RhoA [2]; de même Vav possède un domaine Dbl-like ainsi que BCR, Ect2 [34], Ost [13] p47.lı [35] ou Tiam-1, ce dernier étant amplifié dans des lignées lymphomateuses à fort potentiel métastatique [36]. Ce domaine d'analogie ne leur confère pas forcément une activité d'échange détectable, mais constitue très vraisemblablement un domaine d'interaction protéine-protéine, impliquant ces oncogènes dans la régulation de la signalisation relayée par les Rho. Par ailleurs, trois facteurs d'échange de Ras, (;RF-Ras, hSOSl et hSOS2, présentent aussi un domaine d'échange pour les Rho, illustrant un lien possible entre les voies de signalisation relayées par Ras et celles passant par les Rho [37].

Ce lien, entre deux voies de signalisation importantes pour la prolifération cellulaire, pourrait être aussi représenté par la protéine p190, coimmunoprécipitée avec la GAP de Ras. Cette protéine possède en effet un domaine de type Rho-GAP stimulant l'activité GTPasique de RhoA, Rac et Cdc42 [1].

\section{Conclusion}

On le voit, une véritable dissection moléculaire des voies de signalisation aboutissant au remodelage cytosque- 


\section{RÉFÉRENCES}

26. Jähner D, Hunter T. The Ras related gene $r h o B$ is an immediate early gene inducible by v-fps, epidermal growth factor, and platelet-derived growth factor in rat fibroblasts. Mol Cell Biol 1991 ; 11 : 3682-90.

27. Avraham H, Weinberg RA. Characterization and expression of the human roH/2 gene product. Mol Cell Biol 1989; 9: 205866.

28. Moscow JA, He R, Gnarra JR, et al. Examination of human tumors for thot mutations. Oncogene $1994 ; 9$ : 189-94.

29. Perona R, Esteve P, Jimenez B, Ballestero RP, Ramon y Cajal S, Lacal JC. Tumorigenic activity of tho genes from Aplysia californica. Oncogene 1993; 8 : 1285-92.

30. Jimenez B, Arends M, Esteve P, et al. Induction of apoptosis in NIH3T3 cells after serum deprivation by overexpression of rhop21, a GTPase protein of the ras superfamily. Oncogene $1995 ; 10: 811-6$.

31. Masuda T, Tanaka K, Nonaka H, Yamochi W, Maeda A, Takai Y. Molecular cloning and characterization of yeast tho GDP dissociation inhibitor. I Biol Chem 1994; 269: 19713-8.

32. Qiu RG, Chen J, Kirn D, McCormick F, Symons M. An essential role for Rac in Ras transformation. Nature $1995 ; 374: 457-9$.

33. De Gunzburg J. Les petites protéines $\mathrm{G}$. méderine/sriences $1992 ; 8$ : 322-3.

34. Miki T, Smith CL, L.ong JE, Eva A, Fleming TP. Oncogene ect2 is related to regulators of small GTP-binding proteins. Nature $1993 ; 362: 462-5$.

35. Toksoz D, Williams DA. Novel human oncogene $l b c$ detected by transfection with distinct homology regions to signal transduction products. Oncogene 1994;9: 621-8.

36. Habets GGM, Scholtes EHM, Zuydgeest $\mathrm{D}$, el al. Identification of an invasion-inducing gene, Tiam-l, that encodes a protein with homology to GDP-CTP exchangers for Rho-like proteins. Cell 1994; 77: 537-49.

37. Chardin P. Protéines Ras et transmission des signaux mitogènes. médecine/sriences 1994; 10: 657-64.

38. Pasteris NG, Cadle A, Logie LJ, et al. Isolation and characterization of the faciogenital dysplasia (Aarskog-Scott syndrome) gene : a putative Rho/Rac guanine nucleotide exchange factor. Cell 1994; 79: 669-78.

39. Chant J, Stowers L. GTPase cascades choreographic cellular behavior: movement, morpheogenesis, and more. Cell lettique impliqué à des phases cruciales de la vie cellulaire se fait jour. Tous les partenaires de ces voies de signalisation ne sont pas encore connus. Que penser en effet de l'apparente redondance entre les trois Rho, A, B et C? La surexpression transitoire de ces protéines aboutit aux mêmes effets phénotypiques alors que leur localisation intracellulaire diffère, ainsi que leur mode d'expression (ubiquitaire et constitutif pour RhoA et RhoC, restreint à certains tissus, inductible et dépendant de la phase pour RhoB) $[6,25]$. Peut-être faut-il voir là le signe d'une régulation beaucoup plus subtile de leur fonction, par titration mutuelle et coordonnée dans le temps de leurs effecteurs respectifs, de leurs facteurs d'échange, ou de leurs (;AP, dans certaines circonstances (stimulation mitogénique ou différenciation), dans certains tissus, à certaines phases du cycle cellulaire.

La caractérisation de plusieurs protéines de régulation permettant le couplage entre la voie de signalisation Ras $\rightarrow$ Raf $\rightarrow$ MAP kinase et la cascade des Rho illustre l'importance de la régulation de l'organisation cytosquelettique en matière d'oncogenèse, et plus largement dans les phénomènes de prolifération et différenciation cellulaires. Mais l'étude de la régulation du cytosquelette par les protéines de la famille Rho pourrait aussi déboucher sur la compréhension plus précise des phénomènes de diapédèse (motilité des cellules inflammatoires ou des cellules métastatiques), de contractilité (muscle lisse), de division, de différenciation cellulaire voire même d'embryogenèse, ainsi que la caractérisation récente du gène de la dysplasie faciogénitale d'Aarskog-Scott vient le rappeler $\left(m / s n^{\circ} 3\right.$, vol. 11 , p. 487): ce gène code en effet pour une protéine contenant un domainefacteur d'échange pour les Rho de type $\mathrm{Dbl}[38]$ !

\section{Summary}

Involvement of Rho GTPases in cytoskeleton regulation : putative role in carcinogenesis

Ras-related Rho GTP-binding proteins (Cdc42Hs, Rac, and Rho proteins) have been implicated in the regulation of actin cytoskeleton and in the assembly of integrin receptors which control the morphology, adhesion, and motility of cells; an actual Rho signalling cascade was shown in fibroblasts Swiss 3T3 cells to regulate the formation of cellular actin structures in response to growth factors. Thus, fillopodia, ruffles, lamellipodia, actin stress fibers and focal adhesions were shown to be controlled in turn and respectively by activated Cdc42Hs, Racl, and Rhol proteins. Recent studies led to the identification of several proteins acting along this signalling pathway by regulating the state of activation of the Rho proteins (proteins with exchange-factor activity), or by interacting with activated GTP-bound Rho proteins as actual effectors of these proteins. Several kinases, of which actual precise function remains unclear, have been suspected to belong to this latter category. A direct involvement of RhoA and RhoB proteins has also been suspected in the progress of cell cycle by interaction with the actomyosin system. But, surprisingly, it is another Rho family member, Rac, that was recently shown to potentiate RasV12 transforming activity in fibroblasts cells. Lastly, many oncogenic proteins with Rho/Rac exhange-factor activity and Dbl domain have been recently characterized. Thus, Tiaml activator of Rac was shown to regulate the invasive capacity of RacV12 expressing cells. These experimental observations illustrate the existence of a new signalling pathway Ras $\rightarrow$ Rac leading to transformation or invasiveness, coordinated with, but parallel to, the classical Ras $\rightarrow$ Raf $\rightarrow$ MAP kinase pathway.

\section{TIRÉS À PART}

G. Zalcman. 